\title{
Numerical analysis of the effect of fiber orientation on hydrostatic buckling behavior of fiber metal composite cylinder
}

\author{
BG Sumana', HN Vidya Sagar', KV Sharma' and M Krishna ${ }^{2}$
}

\begin{abstract}
The external hydrostatic buckling behavior of fiber metal laminate (FML) composite cylinders was investigated numerically. The critical buckling pressure predicted by eigenvalue analysis was compared with experimental results. The numerical results showed different modes of buckling and buckling deformation for cylinders of different fiber orientation when subjected to external hydrostatic loading. FML cylinder with $0^{\circ} / 90^{\circ}$ fiber orientation exhibited higher buckling strength and lower buckling deformation as compared to $\mathrm{FML}$ cylinders of $60^{\circ} / 30^{\circ}, \pm 45^{\circ}$, and $\pm 55^{\circ}$ fiber orientations. The orientation of fiber has significant influence on the performance of FML composite cylinder as compared to fiberreinforced plastic thickness. The correlation between numerical and experimental results is discussed in terms of buckling strength, circumferential stiffness, and buckling deformations. It was observed that the cylinders were less sensitive to initial imperfections irrespective of fiber-reinforced plastic thickness. In addition, the results of finite element analysis and experimental results indicate good matches.
\end{abstract}

\section{Keywords}

Fiber metal laminate cylinder, fiber orientation, external hydrostatic loading, eigenvalue buckling, ANSYS

\section{Introduction}

Fiber metal laminates (FMLs) are slowly dominating various fields such as aerospace, marine, and transportation industries due to its higher specific strength and inertness in various environments. Because of lightweight and high resistance to sea water they are used in underwater applications such as unmanned vehicles, ${ }^{1}$ underwater transportation, ${ }^{2}$ etc. In these applications, buckling was a dominant failure due to the compressive stresses exerted by external hydrostatic pressure. ${ }^{3}$ The buckling and postbuckling behaviors of FML cylinders subjected to external hydrostatic pressure have been widely studied using various methods, such as experimental, ${ }^{4}$ mathematical formulation, ${ }^{5}$ and finite element (FE) analysis method. ${ }^{6}$ A few researchers have worked towards comparing the results obtained by different methods. ${ }^{1,3,6,7}$ However, there were constraints to the pattern of failure, mode shape, and forms of distortions that were modeled analytically. Also, very large number of input parameters such as lamina properties, orientations and number of layers, as well as all the geometric and loading parameters associated with isotropic homogeneous cylinders prohibits a pure experimental approach. $^{7}$ In FML composite cylinders, buckling strength was influenced by many parameters such as ply orientation, fiber resin ratio, layer thickness, etc. ${ }^{8}$ To investigate the influence of these parameters through experimental methods was too cumbersome and expensive. Hence, more emphasis was placed on quasi-static numerical simulation of buckling. ${ }^{7}$

Of the many design variables influencing buckling, the thickness and ply orientation were optimized to increase the buckling strength of composite cylinders using response surface method (RSM) of ANSYS. ${ }^{9}$

\footnotetext{
'Department of Mechanical Engineering, University Visvesvaraya College of Engineering, India

${ }^{2}$ Department of Mechanical Engineering, R V College of Engineering, India

\section{Corresponding author:}

BG Sumana, Department of Mechanical Engineering, University Visvesvaraya college of Engineering, K R Circle, Bangalore, 56000I, India. Email: sumogh76.gec@gmail.com
} 


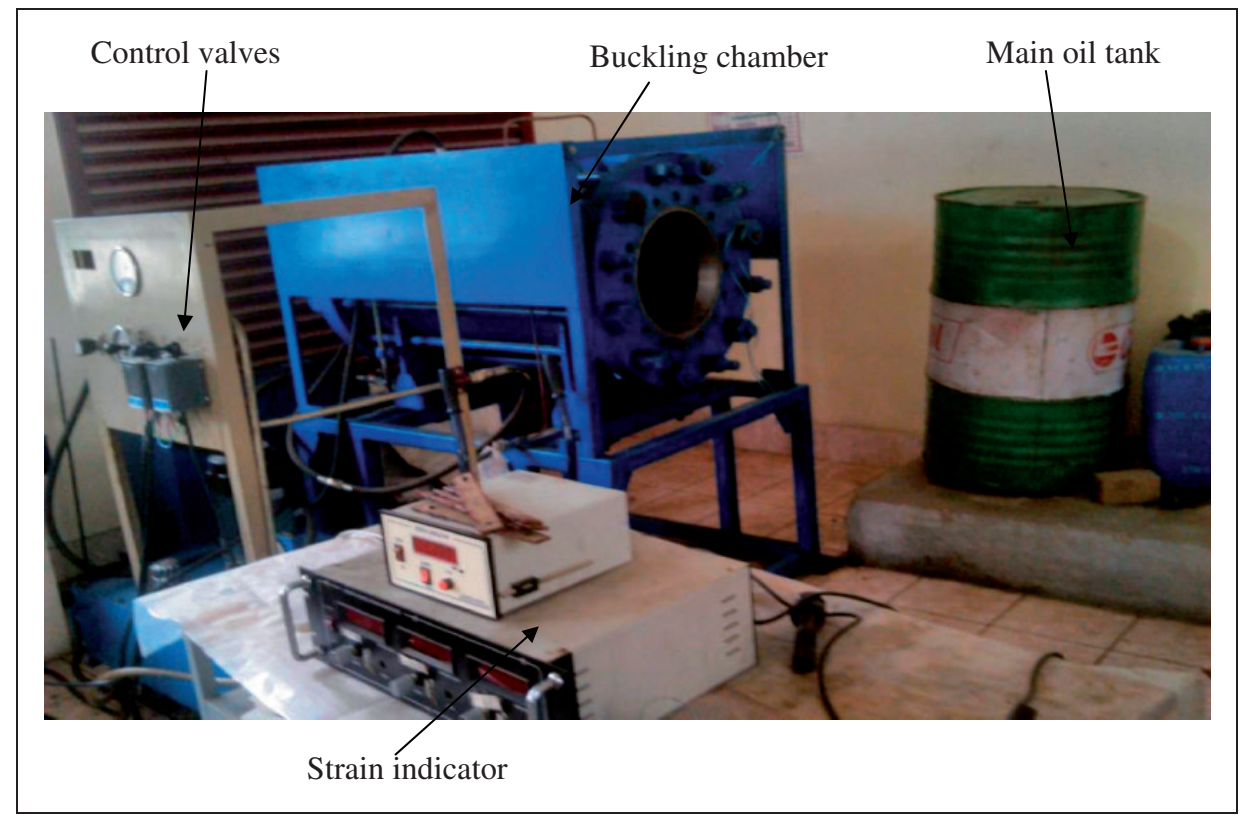

Figure I. Experimental setup to test buckling due to hydrostatic pressure in composite cylinders.

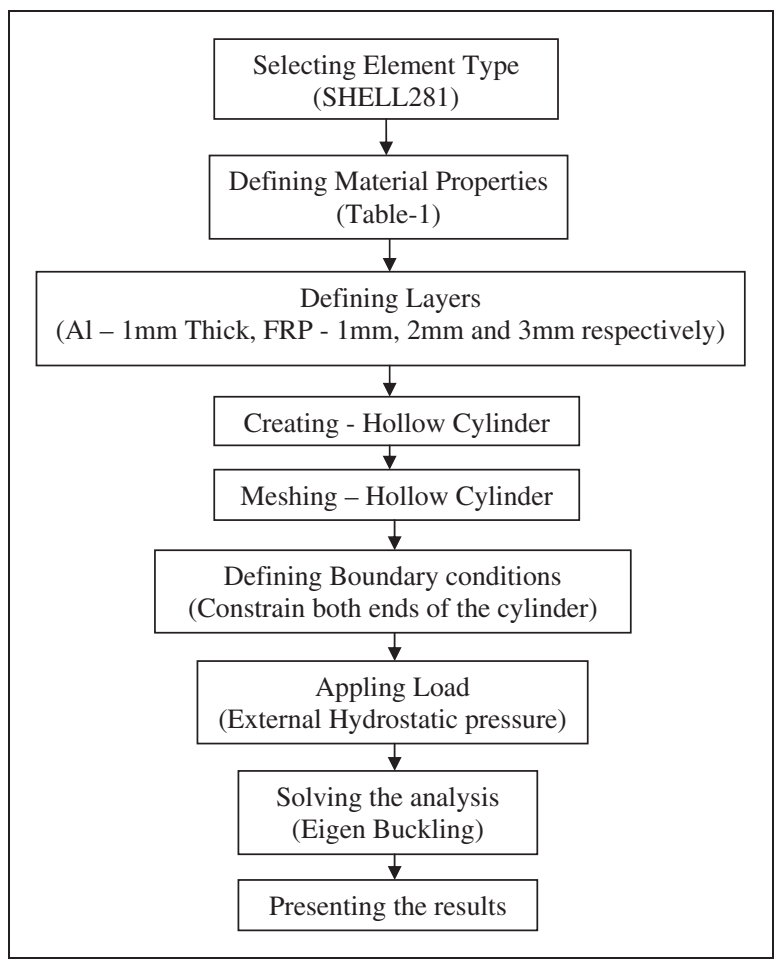

Figure 2. Flow chart of numerical analysis.

Uncertainties of material properties for critical buckling pressure computed by means of Monte Carlo simulation (MCS) and RSM of ANSYS probabilistic design system were compared with experimental data. ${ }^{3}$ Stacking sequence, another significant factor affecting
Table I. Material properties of aluminum (Al 606I-T6) and glass/epoxy composite.

\begin{tabular}{ll}
\hline Aluminum & Value \\
\hline Young's modulus & $70 \mathrm{GPa}$ \\
Poisson's ratio & 0.33 \\
Specific density & $2.7 \mathrm{~g} / \mathrm{cc}$ \\
Glass/Epoxy composite & Value \\
\hline$E_{1}$ & $53.48 \mathrm{GPa}$ \\
$E_{2}, E_{3}$ & $17.7 \mathrm{GPa}$ \\
$v_{12}$ & 0.278 \\
$v_{23}$ & 0.4 \\
$v_{13}$ & 0.278 \\
$G_{12}, G_{23}$ & 5.83 \\
$G_{13}$ & 5.78 \\
Density & $2.1 \mathrm{~g} / \mathrm{cc}$ \\
\hline
\end{tabular}

buckling pressure, was optimized by a genetic algorithm confirmed with finite element method (FEM) results. ${ }^{10}$ Collapse pressure being the end stage of buckling behavior controlled by many variables such as thickness variation and geometrical imperfections was investigated both by experimental and numerical methods. ${ }^{2}$ Tsouvalis et al. ${ }^{8}$ numerically investigated the critical buckling behavior of hydrostatically pressurized mid-span of the composite cylinder along with FE convergence approaches. Buckling was mainly influenced by geometric imperfections such as out-of-roundness 


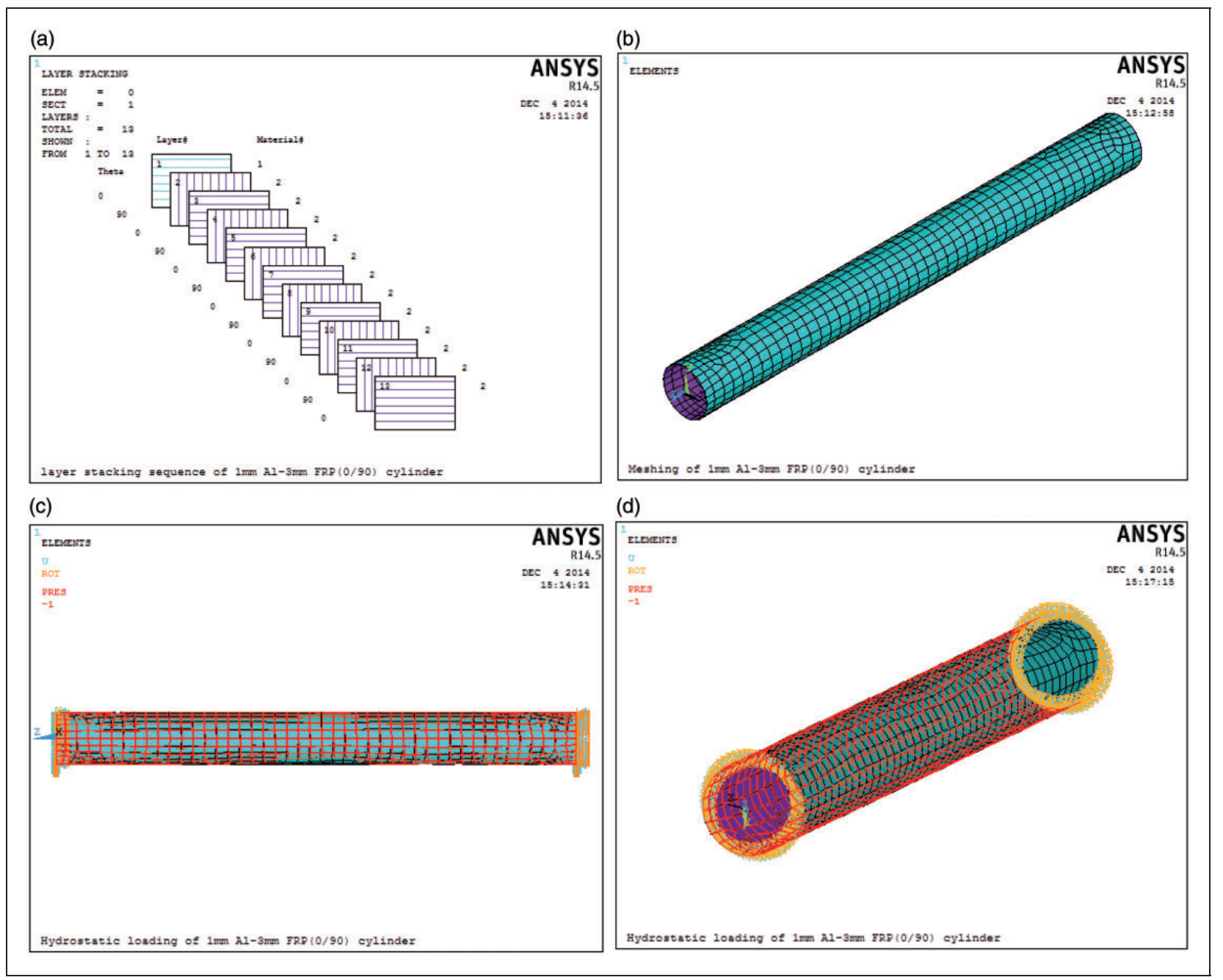

Figure 3. Finite element model: (a) stacking sequence; (b) meshing; (c) boundary conditions; (d) external hydrostatic loading.

and thickness variation generated due to excess resin during manufacturing. But these influences do not affect significantly the performance of composite cylinders subject to external pressure. ${ }^{2}$ Few more researchers numerically investigated composite cylinders subjected to destabilizing loads, ${ }^{11}$ combined external pressure and axial compression, ${ }^{12}$ varying external pressure loads, ${ }^{13}$ buckling and post buckling analysis under hydrostatic pressure. $^{14}$

From these literatures, it can be seen that the FE analysis method carried out on hydrostatic buckling studies was particularly concentrated on isotropic materials and orthotropic cylinders using linear buckling load. Limited amount of research is focused to study buckling analysis of FML cylinders using eigen buckling method. The evaluation of eigenvalue buckling load is a fundamental step to have a first estimate of the strength of the cylinder and may constitute the basis for a design procedure. ${ }^{2}$ Despite the relatively widespread attention given to the problem of buckling in laminated cylinders, there is hardly any information available on the effect of fiber orientation on buckling behavior of a FML cylindrical structure under external hydrostatic loading. This fact motivates the investigation of the present study. The novelty of this research work was to investigate the optimization of fiber orientation on hydrostatic buckling behavior of fiber metal cylindrical structures of different FRP thicknesses.

\section{Experimental studies}

The FML composite cylinder is a cylinder with aluminum metallic liner of inner diameter $80 \mathrm{~mm}, 1 \mathrm{~mm}$ thick, and $800 \mathrm{~mm}$ length on which glass fibers reinforced with epoxy resin was wound at different orientations and thicknesses. Using filament winding machine, a total of twelve composite cylinders were prepared with fibers wound at $0^{\circ} / 90^{\circ}, 60^{\circ} / 30^{\circ}, \pm 45^{\circ}$, 


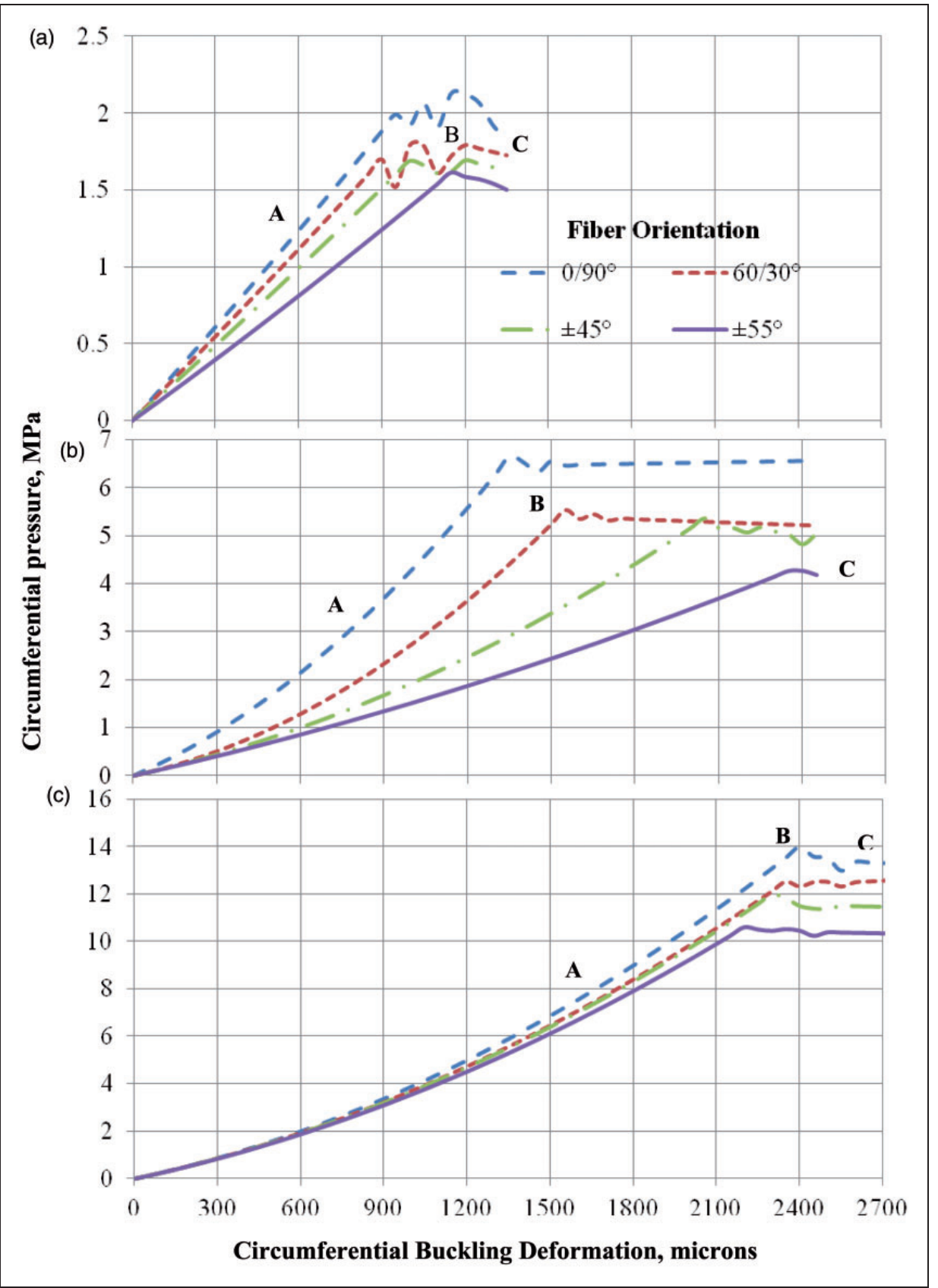

Figure 4. Effect of fiber orientation and FRP thickness on circumferential pressure as a function of buckling deformation of FML composite cylinder: (a) thickness of the cylinder wall Al - I mm and FRP - I mm; (b) thickness of the cylinder wall Al - I mm and FRP - $2 \mathrm{~mm}$; (c) thickness of the cylinder wall Al - I mm and FRP $-3 \mathrm{~mm}$.

and $\pm 55^{\circ}$ orientations and thickness of FRP maintained at $1 \mathrm{~mm}, 2 \mathrm{~mm}$, and $3 \mathrm{~mm}$. The cured FML cylinders were subjected to hydrostatic pressure using an in-house buckling tester which is shown in Figure 1. The testing chamber can apply pressure upto $30 \mathrm{MPa}$, which is equal to the pressure at a depth of $3000 \mathrm{~m}$ in seawater. A high-pressure pump was used to supply hydrostatic pressure. The applied external pressure was increased in steps of $0.2 \mathrm{MPa}$ till failure occurred due to buckling. Strain gages mounted along the axis and circumference was used to measure the buckling deformation.

\section{Numerical solution}

Experimental studies were conducted to investigate the buckling behavior of FML composite cylinders with $0^{\circ} / 90^{\circ}, 60^{\circ} / 30^{\circ}, \pm 45^{\circ}$, and $\pm 55^{\circ}$ fiber orientations and 


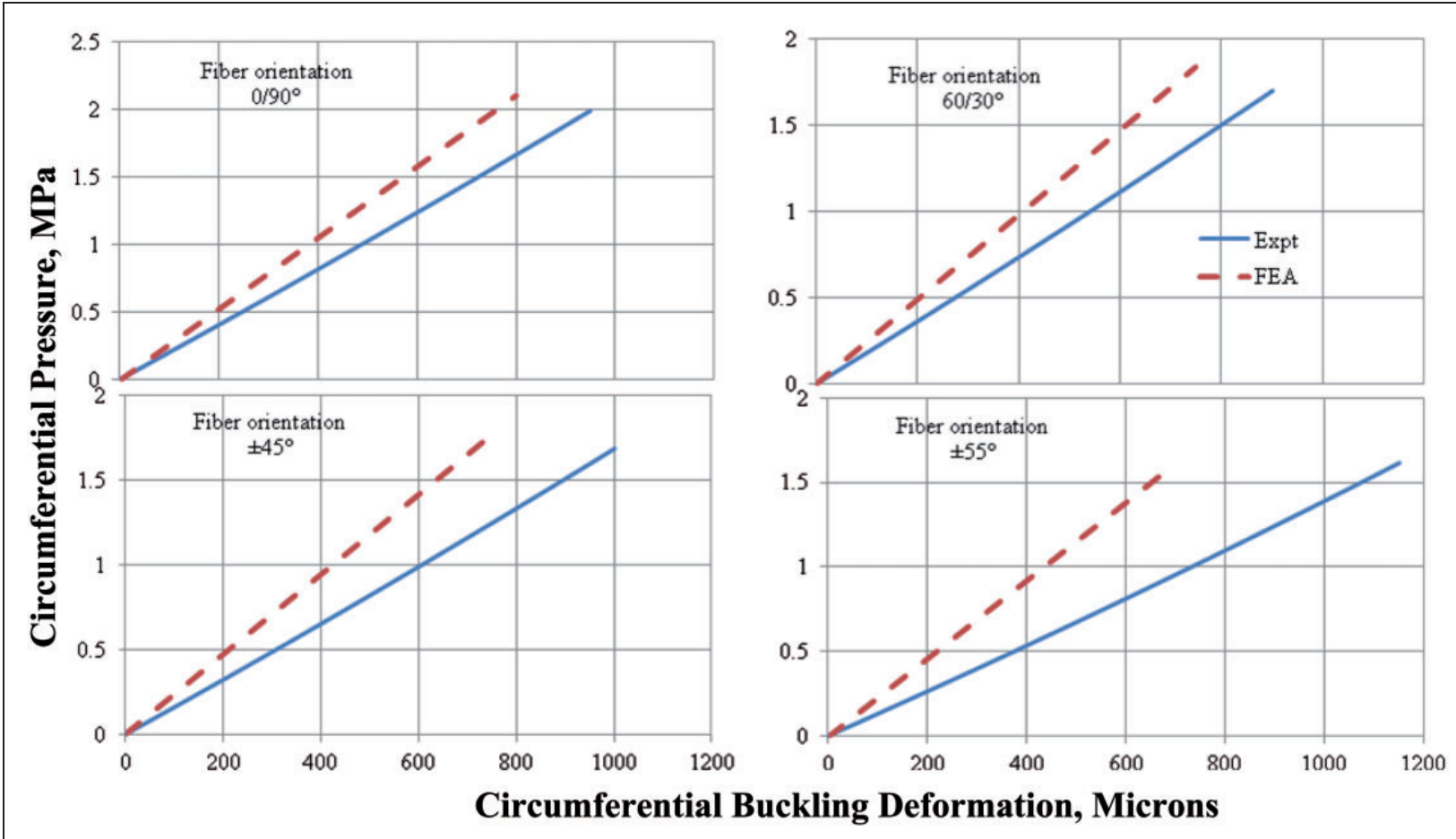

Figure 5. Comparison of the effect of circumferential pressure on pre-buckling deformation of FML composite cylinders of I mm FRP thickness.

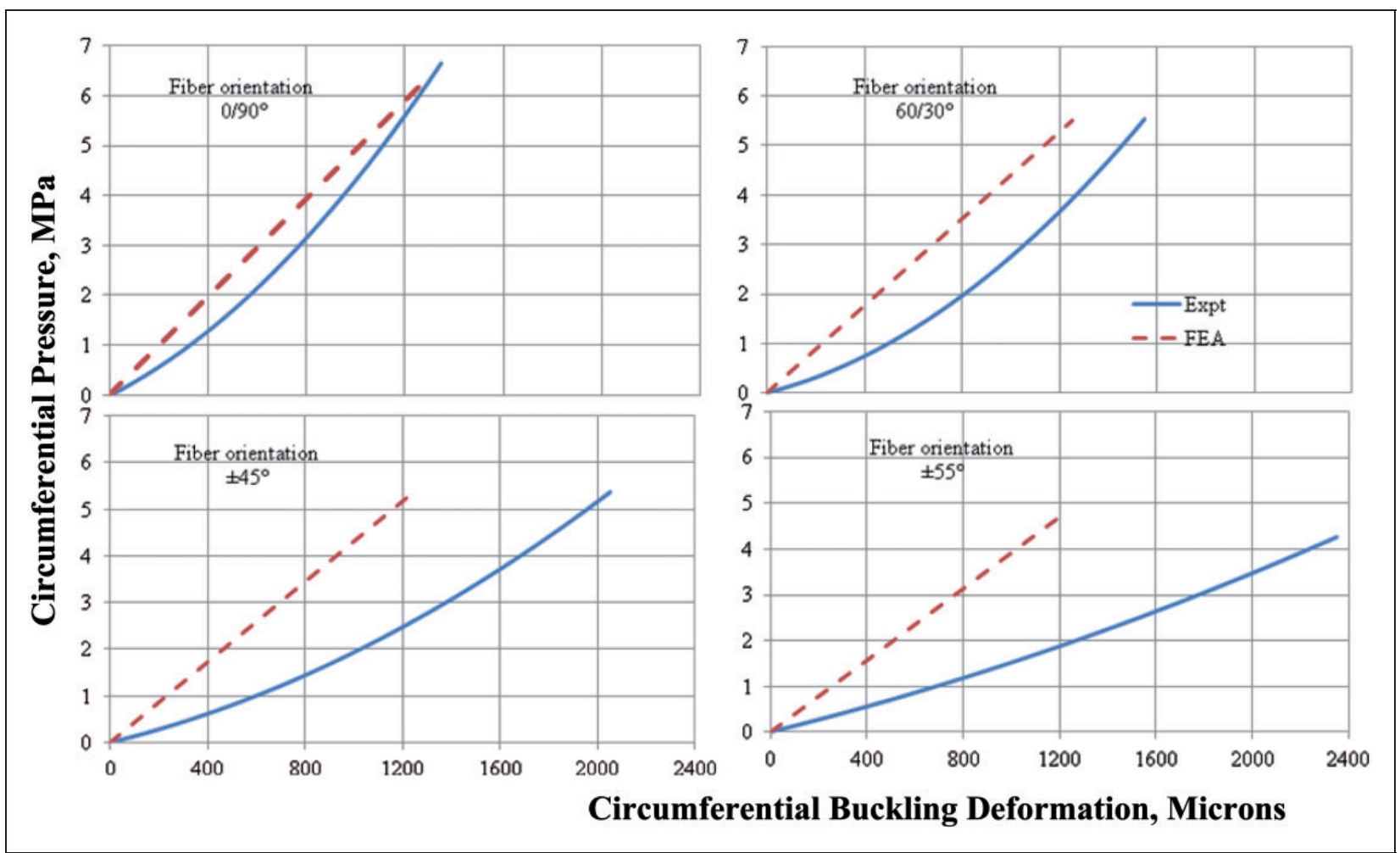

Figure 6. Comparison of the effect of circumferential pressure on pre-buckling deformation of FML composite cylinders of $2 \mathrm{~mm}$ FRP thickness. 


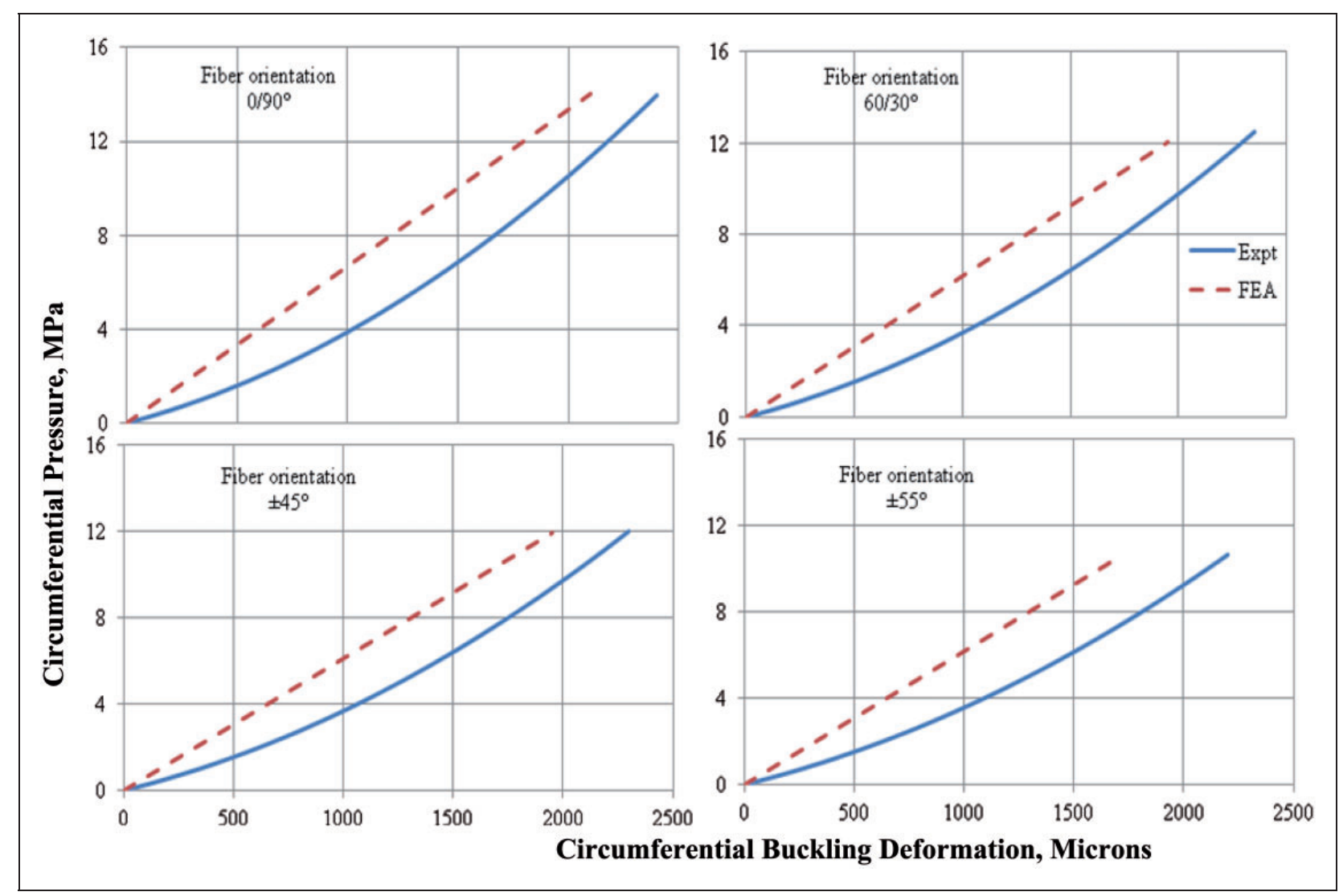

Figure 7. Comparison of the effect of circumferential pressure on pre-buckling deformation of FML composite cylinders of $3 \mathrm{~mm}$ FRP thickness.

thicknesses of FRP as $1 \mathrm{~mm}, 2 \mathrm{~mm}$, and $3 \mathrm{~mm}$. To validate the previously obtained experimental results and to determine the factors influencing buckling, numerical approach is necessary for a given material, geometry, and loading conditions. Simulation has been carried out by using ANSYS 14.5 FE package. The flow chart shown in Figure 2 describes the steps involved in using the software for eigen buckling analysis of FML composite cylinder.

A detailed description of the steps involved in using the software for analysis of FML composite cylinder is described in the following section.

\section{Element type and material properties}

The element type used was SHELL281 for both Al metallic liner and FRP. SHELL281 is suitable for analyzing moderately thick shell structures. It has eight nodes with six degrees of freedom at each node: translations in the $x-, y$-, and $z$-axes, and rotations about the $x$-, $y$-, and $z$-axes. This element may be used for layered applications for modeling composite shell structures. The mechanical properties needed are modulus of elasticity $(E)$ and Poisson's ratio $(v)$ for linear isotropic material i.e. aluminum and orthotropic properties for
FRP. The mechanical properties of Al 6061 and glassepoxy composite material are listed in Table 1.

\section{Modeling and meshing}

Aluminum metallic liner of $1 \mathrm{~mm}$ thickness is input as one layer, on which FRP of $0.25 \mathrm{~mm}$ thickness with respective winding angle is considered for the FE analysis. The layer stacking sequence of $3 \mathrm{~mm}$ FRP wound on $1 \mathrm{~mm}$ Al metallic liner is shown schematically in Figure 3(a). A hollow cylinder was created using the option of solid cylinder and then deleting the volume and areas. This was done in order to maintain same displacement of the interface. The composite cylinder was then meshed as shown in Figure 3(b). The maximum number of nodes was 3145 and maximum deformation was observed around node 1208 .

\section{Constraints and loading}

The ends of the composite cylinder were constrained along all degrees of freedom, as shown in Figure 3(c). An external pressure of $p=-1$ was applied on the external surfaces of composite as shown in Figure 3(d). Static analysis and eigen buckling analysis 


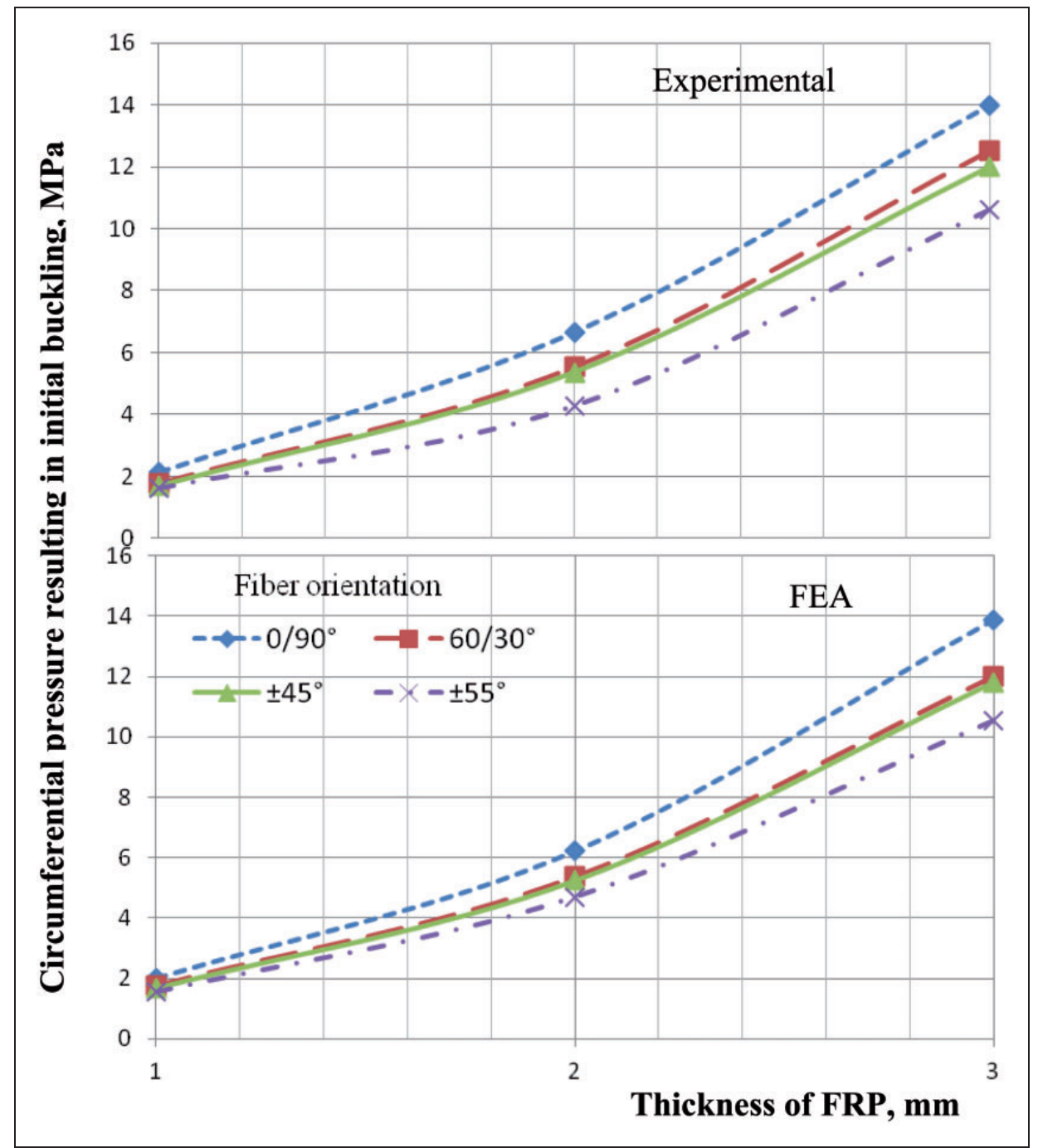

Figure 8. Comparison of the effect of fiber orientation on circumferential pressure (causing initial buckling) as a function of FRP thickness of FML composite cylinder.

Table 2. Comparison of experimental and numerical values of buckling pressure for different thicknesses.

\begin{tabular}{|c|c|c|c|c|c|c|c|c|c|}
\hline & \multicolumn{3}{|l|}{$\mathrm{I} \mathrm{mm}$} & \multicolumn{3}{|l|}{$2 \mathrm{~mm}$} & \multicolumn{3}{|l|}{$3 \mathrm{~mm}$} \\
\hline & Expt & FEA & \% Deviation & Expt & FEA & \% Deviation & Expt & FEA & \% Deviation \\
\hline $0 / 90^{\circ}$ & 2.1268 & 2.0039 & 6.13 & 6.6555 & 6.2182 & 7.03 & 13.9949 & 13.885 & 0.79 \\
\hline $60^{\circ} / 30^{\circ}$ & 1.7939 & 1.7654 & 1.61 & 5.5413 & 5.3768 & 3.05 & 12.5092 & 12.013 & 4.13 \\
\hline $\pm 45^{\circ}$ & 1.6939 & 1.6919 & 0.11 & 5.3597 & 5.239 & 2.30 & 11.9832 & 11.783 & 1.69 \\
\hline $\pm 55^{\circ}$ & 1.6140 & 1.5473 & 4.31 & 4.2720 & $4.698 I$ & 9.06 & 10.6018 & $|0.54|$ & 0.57 \\
\hline
\end{tabular}

FEA: finite element analysis.

using Block Lancos extraction method were performed in sequence to obtain the critical buckling pressure. Static buckling was performed to determine the prebuckling deformation. The linear eigenvalue buckling analysis was used to determine buckling load - initial/ critical load at which a structure becomes unstable and buckled mode shapes - the characteristic shape associated with a structure's buckled response. ${ }^{15}$ 


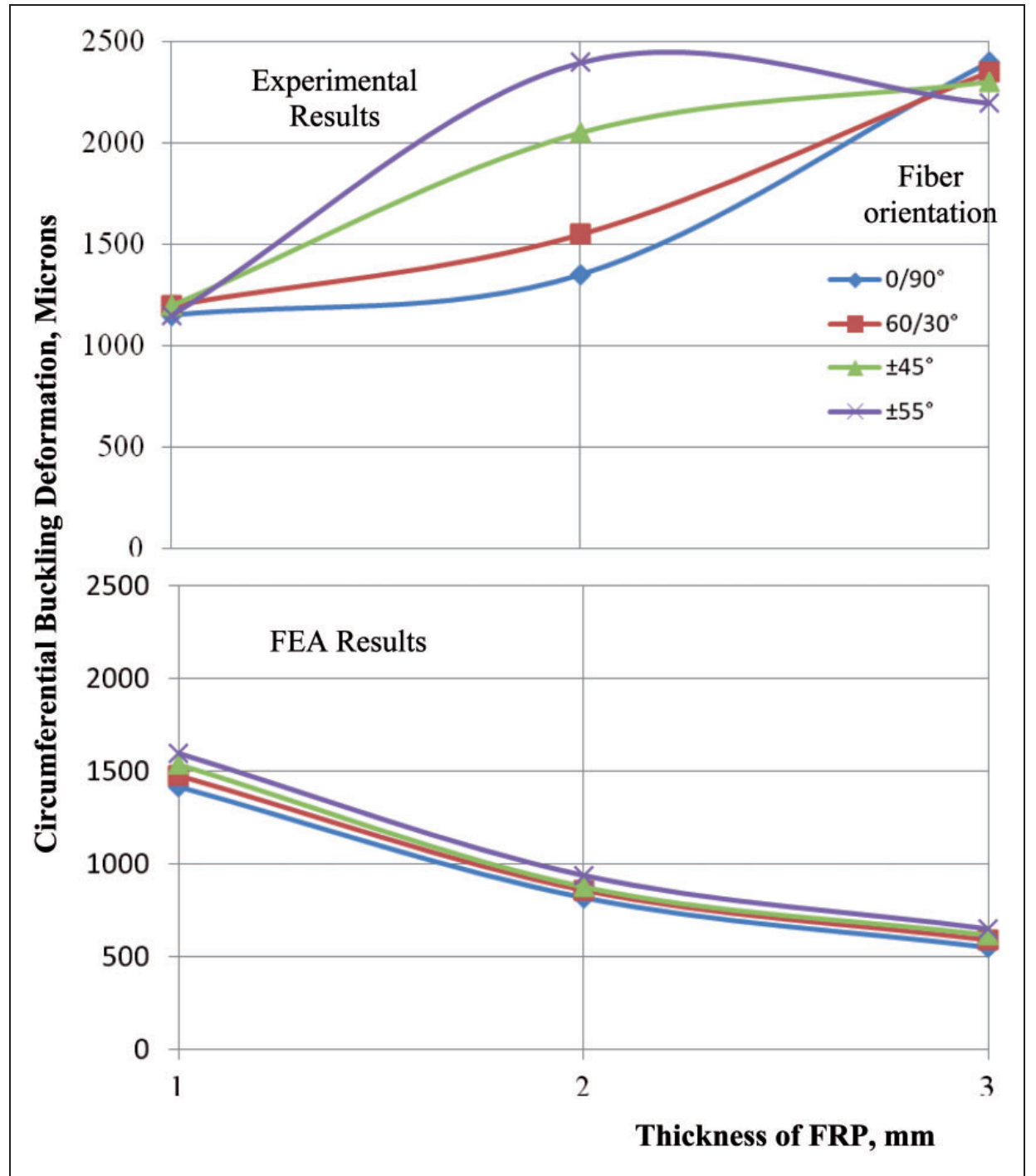

Figure 9. Comparison of the effect of fiber orientation on circumferential buckling deformation as a function of FRP thickness of FML composite cylinder.

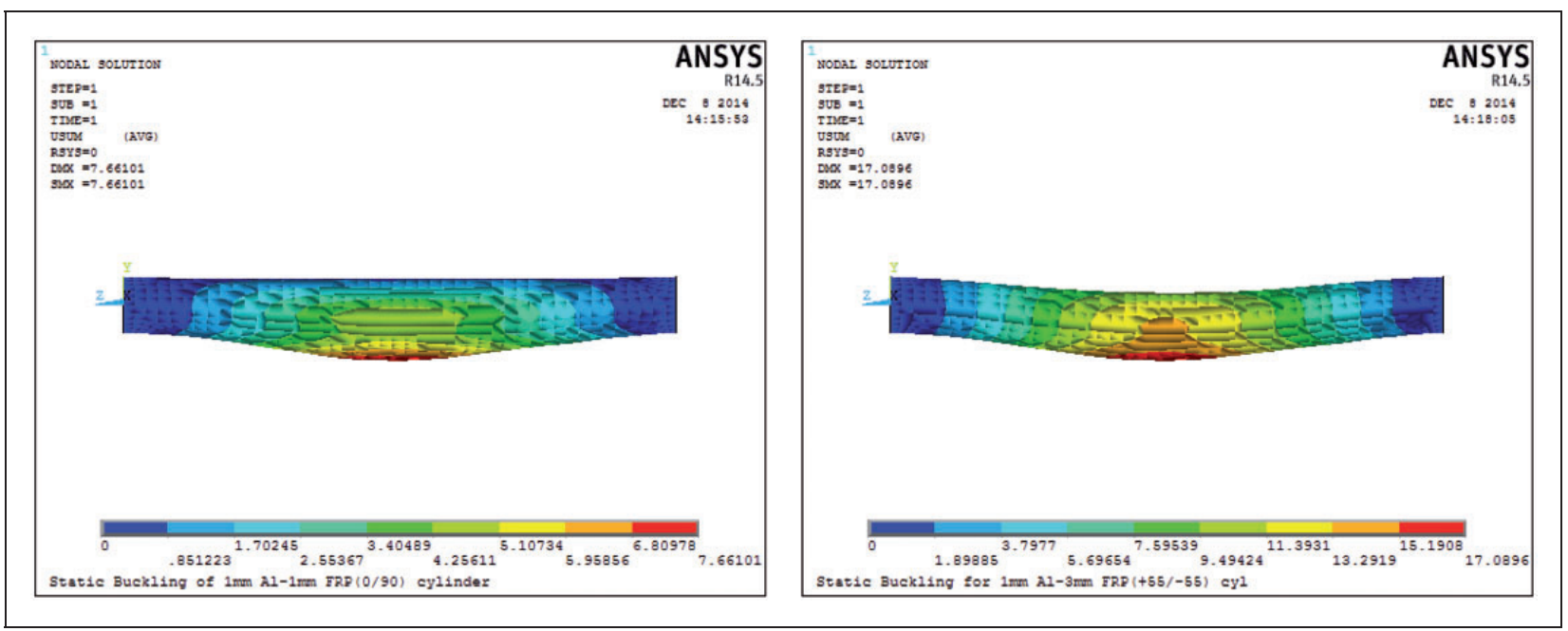

Figure 10. Static buckling modes of FML composite cylinder with $0 / 90^{\circ}$ and $\pm 55^{\circ}$ fiber orientation. 


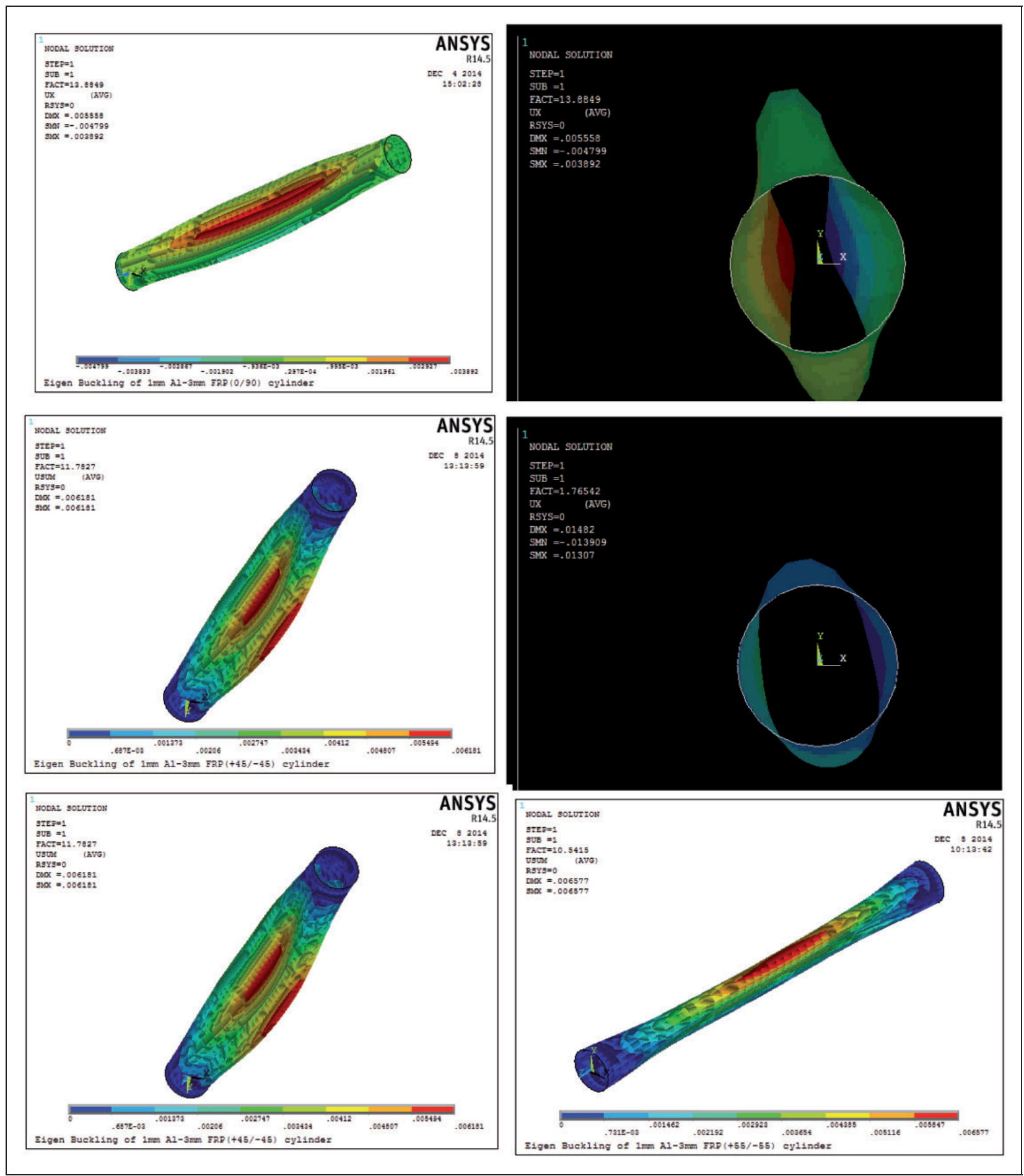

Figure II. Buckling modes of FML composite cylinder of different fiber orientation.

It only predicts the pre-buckling deformation, but does not solve the magnitude of deformation post buckling. ${ }^{16}$ The results for all the composite cylinders with its respective orientation and thickness are discussed in the following section.

\section{Results and discussion}

Composite cylinders of $0^{\circ} / 90^{\circ}, 60^{\circ} / 30^{\circ}, \pm 45^{\circ}$, and $\pm 55^{\circ}$ fiber orientations and thicknesses of FRP $1 \mathrm{~mm}, 2 \mathrm{~mm}$, and $3 \mathrm{~mm}$ exhibited buckling when subjected to external hydrostatic pressure. 
Figure 4 shows the experimental values of the circumferential hydrostatic pressure and its corresponding buckling deformation. FML cylinders of considered fiber orientations showed three regions of behavior. Linear increasing circumferential pressure in region "A" resulted in increasing deformation up to a peak value. At peak, buckling was initiated and thereafter zig-zag pattern of consecutive buckling was observed in region " $\mathrm{B}$ " and then constant deformation with decreased circumferential pressure in region " $\mathrm{C}$ ".

The experimental results discussed above were validated numerically using linear eigenvalue analysis. The static analysis investigated the pre-buckling behavior and compared with the experimental results as shown in Figures 5 to 7 for FRP thickness of $1 \mathrm{~mm}, 2 \mathrm{~mm}$, and $3 \mathrm{~mm}$, respectively. For a FML composite cylinder of $1 \mathrm{~mm}$ FRP thickness as shown in Figure 5, the FEA result showed that the pre-buckling curve indicating deformation due to circumferential pressure increased linearly, while the experimental result showed that the curve increased exponentially with power of $n=1.2$. The results of each composite cylinder were analyzed to fit them into polynomial curves. The FEA results indicated linearity with adjusted $R^{2}=1$, while the adjusted $R^{2}$ was 0.99 for experimental results, indicating no significant deviation of the buckling pressure.

Similarly, for a FML composite cylinder of $2 \mathrm{~mm}$ and $3 \mathrm{~mm}$ FRP thickness as shown in Figures 6 and 7 respectively, the FEA results showed that the curve increased linearly, while the experimental result showed that the curve increased exponentially with power of $n=1.44$ and 1.42 , respectively. These results were analyzed to fit them into polynomial curves. The FEA results indicated linearity with adjusted $R^{2}=1$, while the best fit for experiment results were obtained in quadratic polynomial curves with $R^{2}=1$. This indicates that the FEA model is stiffer that the experimental model as it disregards details such as geometric and material imperfections ${ }^{17}$ and hence the cylinders were less sensitive to initial imperfections. ${ }^{18}$

The experimental results of the external hydrostatic circumferential pressure as it varies with fiber orientation due to the effect of FRP thickness were compared with FEA results and plotted in Figure 8. The experimental results and FE predictions listed in Table 2 are within a deviation of $10 \%$. This was considered satisfactory due to possible geometric, material, and loading uncertainties between physical tests and numerical simulation. ${ }^{7}$ Also, certain types and magnitudes of small initial geometric imperfections can have a stiffening effect on the buckling behavior of FML composite cylinders. ${ }^{8}$

The experimental and numerical results of the effect of fiber orientation on circumferential buckling deformation as a function of FRP thickness are plotted in
Figure 9. It can be observed from the experiemntal results, that the buckling deformation of 2-mm-thick cylinder is spread over a wide value unlike FML cylinders of $1 \mathrm{~mm}$ and $3 \mathrm{~mm}$ FRP thicknesses, which lie within a closed range. From the results, it is evident that FML cylinder with $0^{\circ} / 90^{\circ}$ fiber orientation possesses highest buckling strength (Figure 7) and least buckling deformation (Figure 9). As the orientation of fiber increases from $0^{\circ}$ to $90^{\circ}$, buckling pressure increases with decrease in buckling deformation, due to stiffening of the cylinder in circumferential direction. ${ }^{9}$

The buckling modes corresponding to FML cylinders of $0^{\circ} / 90^{\circ}, 60 / 30^{\circ}, \pm 45^{\circ}$, and $\pm 55^{\circ}$ fiber orientation with FRP thickness of $3 \mathrm{~mm}$ are shown in Figures 10 and 11. The snapshots of the buckling behavior presented explain the physical phenomena occurring during the analysis. Pre-buckling as shown in Figure 10 is characterized by barrelling with small buckles which grow substantially as the buckling pressure is reached. The outward bulge developed during pre-buckling changes to inward buckling at the point of critical buckling pressure ${ }^{7}$ as shown in Figure 11. The failure mode of the FML composite cylinders was consistently an elliptical cylindrical two-wave shape with maximum stress and maximum deformation towards the center of the cylinder. This type of failure proves that there was no radial restraint on the constrained edges of the cylinder. ${ }^{19}$

\section{Conclusion}

From the numerical analysis performed to investigate the buckling behavior of FML composite cylinders with $0^{\circ} / 90^{\circ}, 60 / 30^{\circ}, \pm 45^{\circ}$, and $\pm 55^{\circ}$ fiber orientation and FRP thicknesses $1 \mathrm{~mm}, 2 \mathrm{~mm}$, and $3 \mathrm{~mm}$, the following conclusion can be summarized:

(i) The prediction of hydrostatic buckling pressure using eigenvalue FE analysis for FML cylinders are close to the experimental values.

(ii) The variation of FRP thickness and fiber orientation influences circumferential pressure, especially in case of external hydrostatic loading.

(iii) The load-shortening curve shows linear increase in pre-buckling and a zig-zag mode at consistent load post buckling.

(iv) Increase of fiber orientation from $0^{\circ}$ to $90^{\circ}$ increased buckling pressure and reduced buckling deformation due to stiffening of the cylinder in circumferential direction.

(v) FML cylinder with $0^{\circ} / 90^{\circ}$ fiber orientation possessed higher buckling strength and lowest buckling deformation followed by FML cylinders of $60^{\circ} / 30^{\circ}, \pm 45^{\circ}$, and $\pm 55^{\circ}$ fiber orientations. 
(vi) Typically the optimum configuration in case of hydrostatic pressure has $0^{\circ} / 90^{\circ}$ fiber orientation in both experimental and numerical studies.

\section{Conflict of interest}

None declared.

\section{Funding}

This research received no specific grant from any funding agency in the public, commercial, or not-for-profit sectors.

\section{References}

1. Moon C-J, Kim I-H, Choi B-H, et al. Buckling of filament-wound composite cylinders subjected to hydrostatic pressure for underwater vehicle applications. Compos Struct 2010; 92: 2241-2251.

2. Carvelli V, Panzeri N and Poggi C. Buckling strength of GFRP under-water vehicles. Compos Part B: Eng 2001; 32: 89-101.

3. Cai BP, Liu YH, Liu ZK, et al. Probabilistic analysis of composite pressure vessel for subsea blowout preventers. Eng Fail Anal 2012; 19: 97-108.

4. Hur S-H, Son H-J, Kweon J-H, et al. Postbuckling of composite cylinders under external hydrostatic pressure. Compos Struct 2008; 86: 114-124.

5. Messager T. Buckling of imperfect laminated cylinders under hydrostatic pressure. Compos Struct 2001; 53: 301-307.

6. Han JY, Jung HY, Cho JR, et al. Buckling analysis and test of composite shells under hydrostatic pressure. J Mater Process Technol 2008; 201: 742-745.

7. Chryssanthopoulos MK, Elghazouli AY and Esong IE. Validation of FE models for buckling analysis of woven GFRP shells. Compos Struct 2000; 49: 355-367.

8. Tsouvalis NG, Zafeiratou AA and Papazoglou VJ. The effect of geometric imperfections on the buckling behaviour of composite laminated cylinders under external hydrostatic pressure. Compos Part B: Eng 2003; 34: 217-226.

9. Goldfeld Y, Arbocz J and Rothwell A. Design and optimization of laminated conical shells for buckling. ThinWall Struct 2005; 43: 107-133.

10. Messager T, Pyrz M and Chauchot P. Optimized laminations for submarine composite hulls. Eur J Comput Mech 2012; 9: 199-215.

11. Han B and Simitses GJ. Analysis of anisotropic laminated cylindrical shells subjected to destabilizing loads. Part II: Numerical results. Compos Struct 1991; 19: 183-205.

12. Li Z-M and Lin Z-Q. Non-linear buckling and postbuckling of shear deformable anisotropic laminated cylindrical shell subjected to varying external pressure loads. Compos Struct 2010; 92: 553-567.

13. Li Z-M and Qiao P. Buckling and postbuckling of anisotropic laminated cylindrical shells under combined external pressure and axial compression in thermal environments. Compos Struct 2015; 119: 709-726.

14. Kasagi A and Sridharan S. Buckling and postbuckling analysis of thick composite cylindrical shells under hydrostatic pressure. Compos Eng 1993; 3: 467-487.

15. Wang X, Xiao J and Zhang YC. A method for solving the buckling problem of a thin-walled shell. Int J Press Vess Pip 2004; 81: 907-912.

16. Chen W-F and Atsuta T. Theory of beams and columns, Vol 1: In-plane behaviour and design. Plantation, FL: J Ross Publishing, 2009.

17. Budgell P. Finite Element Analysis: Modeling Issues and Ideas, 2004. http://home.cogeco.ca/ pbudgell/ Modeling_issues.html.

18. Shen H-S and Xiang Y. Buckling and postbuckling of anisotropic laminated cylindrical shells under combined axial compression and torsion. Compos Struct 2008; 84: 375-386.

19. Hubner A, Albiez M, Kohler D, et al. Buckling of long steel cylindrical shells subjected to external pressure. Thin-Wall Struct 2007; 45: 1-7. 